\title{
Laser Performance of a PLD-Grown Yb:LuAG Double-Clad Planar Waveguide
}

\author{
Sergey V. Kurilchik, Jake J. Prentice, Robert W. Eason, and Jacob I. Mackenzie \\ Optoelectronics Research Centre, University of Southampton, University Road, Southampton, SO17 1BJ, UK
}

A growing demand for compact high-power laser sources has recently stimulated significant interest in the development of planar waveguide laser geometries compatible with high-power laser diode bars and producing high beam quality laser output [1,2]. This can be achieved by use of cladding-pumping techniques such that the pump and the laser signals are guided by different, but spatially overlapping, waveguides. By engineering the doped region to be the central layer of a double-clad planar waveguide, fundamental-mode selection can be achieved [1]. Fabrication of these structures is possible using pulsed laser deposition (PLD), which combines relatively high growth rates (up to $25-\mu \mathrm{m}$ per hour [3]), the ability to dynamically control local refractive index [4], and the capability to grow active hetero-epitaxial multi-layered structures [5].

In this work we present a $4 \mu \mathrm{m}$-thick 7 at.\% Yb-doped LuAG core layer bounded by two $2-\mu \mathrm{m}$-thick undoped LuAG cladding layers, which was grown on a YAG substrate by use of PLD. X-ray diffraction analysis confirmed a film with predominantly $<100>$ crystal orientation. Absorption and stimulated emission cross sections were calculated using the measured excited state lifetime and fluorescence spectrum, which compares well with previously reported spectroscopy of $\mathrm{Yb}$ :LuAG-crystals grown by traditional methods. An upper limit for the propagation losses of the waveguide were estimated to be $1.2 \mathrm{~dB} \cdot \mathrm{cm}^{-1}$ by measurement of the transmission of a $1064 \mathrm{~nm}$ laser beam focussed into the end facet of the waveguide.

Laser experiments were carried out with a quasi-monolithic plane-plane cavity shown schematically in Fig.1(a). A conduction-cooled $40 \mathrm{~W}$ diode-laser bar emitting at $940 \mathrm{~nm}$ wavelength, with fast- and slow-axis collimation (FAC/SAC), was used as a pump source. Lasing was obtained for both simple Fresnel reflection and for output couplers with reflectance of $30 \%$ or $50 \%$ (M2), the measured performance is illustrated in Fig. 1(b).

(a)

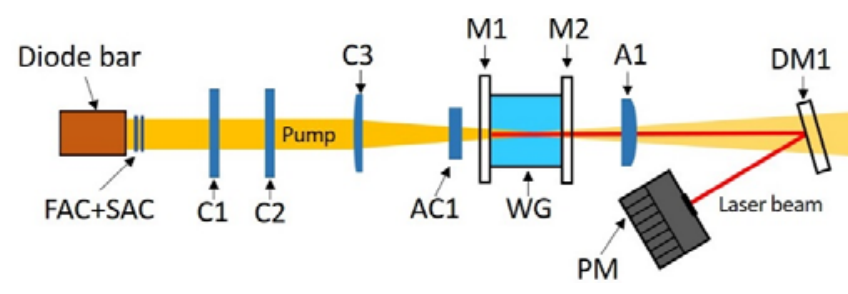

(b)

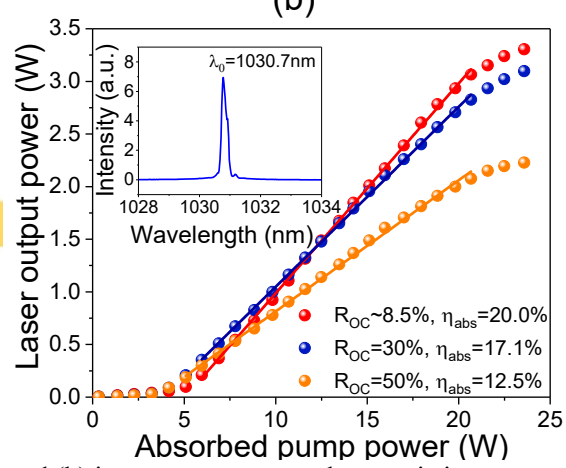

Fig. 1 Yb:LuAG double-clad waveguide laser (a) experimental setup and (b) input-output power characteristics

The best laser performance was achieved with a high-reflectance mirror (M1) and Fresnel reflection at the output facet giving a maximum output power of $3.3 \mathrm{~W}$ at $1030.7 \mathrm{~nm}$ with a $20 \%$ slope efficiency. This is the first demonstration to our knowledge of a $\mathrm{Yb}: \mathrm{LuAG}$ crystalline planar waveguide laser, which has a slope efficiency of more than twice that reported previously for a ceramic $\mathrm{Yb}: \mathrm{LuAG}$ waveguide laser [6]. The measured laser spectrum is displayed in Fig.1(b) as an insert. Thermally induced roll-over of the lasing output was noticeable at pump power levels above $20 \mathrm{~W}$, which is expected to be mitigated with better heat-sinking. Using a modified Caird analysis for the case of large output coupler transmission and losses, the single pass parasitic loss in the cavity was calculated to be $\sim 1.8 \mathrm{~dB}$. Improved laser performance and the beam quality is expected with further growth optimisation, which will be enabling for the construction of efficient high-power lasers and amplifiers.

\section{References}

[1] T. Bhutta, J. I. Mackenzie, D. P. Shepherd, R. J. Beach, "Spatial Dopant Profiles For Transverse-Mode Selection in Multi-Mode Waveguides,"J. Opt. Soc. Am. B 19, 1539-1543 (2002).

[2] J. I. Mackenzie, J. W. Szela, S. J. Beecher, T. L. Parsonage, R. W. Eason and D. P. Shepherd, "Crystal Planar Waveguides, a Power Scaling Architecture for Low-Gain Transitions," IEEE J. Sel. Top. Quantum Electron. 21(1), 380-389 (2015).

[3] J. Mackenzie, J. Prentice, J. Grant-Jacob, S. Kurilchik, D. Shepherd, and R. Eason, "Functional crystal films fabricated by pulsed laser deposition," presented at PHOTONICS International Congress, Yokohama, Japan 23-27 Apr. 2018.

[4] J. A. Grant-Jacob, S. J. Beecher, H. Riris, A. W. Yu, D. P. Shepherd, R. W. Eason, and J. I. Mackenzie, "Dynamic control of refractive index during pulsed-laser-deposited waveguide growth,” Opt. Mater. Express 7, 4073-4081 (2017).

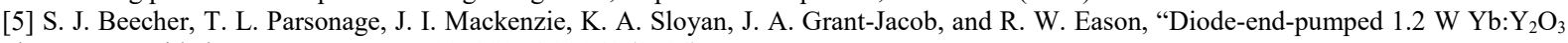
planar waveguide laser," Opt. Express 22, 22056-22061 (2014).

[6] C. Ma, J. Zhu, K. Liu, F. Tang, J. Long, Z. Wen, R. Ma, X. Yuan, W. Guo, J. Li, and Y. Cao, "Longitudinally diode-pumped planar waveguide YAG/Yb:LuAG/YAG ceramic laser at 1030.7 nm,” Opt. Lett. 41, 3317-3319 (2016). 